\title{
Comparing the Psychological Well-Being of the Manjo Ethnic Minority with the Non-Manjo Ethnic Group in Kaffa Zone, Ethiopia.
}

\author{
Bizuayehu Dengechi ${ }^{1}$ \\ MizanTepi University, Ethiopia \\ Nigatuwa Worku \\ Jimma University, Ethiopia \\ Fisseha Mikre \\ Jimma University, Ethiopia
}

\begin{abstract}
This study examined the level of psychological well-being between the Ethnic Minority group, commonly called "Manjo," and the majority group called "Gomero." Psychological well-being questionnaires were administered to a sample of 298 (independent sample from both groups). The findings demonstrated that the nonManjo (Gomero) Ethnic group possessed a considerably high level of psychological well-being. Statistical differences were found in participants' psychological well-being across Ethnic groups. According to the results, participants from the Manjo Ethnic Minority group had a lower level of psychological well-being $(\mathrm{M}=211.27, \mathrm{SD}=17.51)$ compared to the majority (Gomero). A statistically significant variation in psychological well-being (theoretically embodied across a broad spectrum of measurement units) among the two independent study groups was reflected.
\end{abstract}

Keywords: ethnic minority, Kaffa, Manjo, psychological well-being.

From existential and humanistic viewpoints, psychological well-being describes engagement in life challenges and may be operationalized with assessments of purpose in life, personal growth, autonomy, environmental mastery, self-acceptance, and positive relations with others (Ryff \& Singer, 2008). According to Ryff (1989), Psychological well-being refers to the extent to which people feel that they have meaningful control over their lives and their activities. Psychological well-being problems have become increasingly common across different social classes nowadays, particularly these ethnic groups who have been labeled as or treated differently from other persons without justification, only because of their biological, physiological or personal characteristics, their origin or language, their abilities, manifestations of belief or preferences. The risks of such experiences are, however, not randomly distributed among the population (Olli \& Olsen, 2006). Some studies have reported that perceived ethnic discrimination is significantly related to high psychological distress levels (Williams, et al., 2009), whereas others have suggested that the relationship is not straightforward.

Hence, Ryff, and Singer (1996), had introduced six core dimensions of psychological wellbeing, explicitly: 1) self-acceptance (state of having positive thoughts and feelings about oneself); 2) positive relations with others (ability to engage in warm and trusting relationship with others); 3) autonomy (ability to be independent and coping with social pressure); 4) environmental mastery

${ }^{1}$ Corresponding Author E-Mail: bizuye006@ gmail.com 
(ability to adapt, change or create one's environment according to one's needs through physical and mental activities); 5) purpose in life (state of having objectives and goals in life and working towards achieving goal-oriented); and 6) personal growth (continuously growing and developing as oneself). This multi-perspective notion of psychological well-being validates the connections between psychological well-being and life esteem as well as life satisfaction (Armsden\& Greenberg, 1987); mindfulness (Brown \& Ryan, 2003); physical activity (Biddle \&Asare, 2011) and social support (Lakey\&Orehek, 2011).

Ethnic minorities like the Fuga, Wayto, and Waata in Ethiopia, who are frequently subject to exclusion, often face psycho-social difficulties in different parts of Ethiopia (Yoshida, 2008). Similarly, in the Kaffa zone, where the current study focused, Manjos are a minority group that has a victim of discrimination because of ethnicity and a long-past historical lifestyle attached to it. The neighboring non-Manjo (Gomero) communities have been unwilling to have social and economic integration with Manjo (Farm Africa, 2002). The Gomero have their own identity as members of the majority ethnic group and live within the majority culture (Yoshida, 2013).Manjo clans were considered to lack self-confidence, be anxious about their surroundings, and have immature intelligence (Mesfin, 2005). Individuals from the Manjo ethnic minority (specifically those who live in semi-urban areas) also experience psychological problems such as a lack of selfconfidence, frustration, mistrust, fear, and a feeling of inferiority (Ahmed, 2009; Ersoy \& Uysal, 2018). The practices of discrimination against Manjo Ethnic Minorities can be manifested through keeping at a distance, psychological inhibition, material distinction, and communication restrictions. Most of these discriminatory actions have prevailed for at least half a century. Studies have shown that poor psychological well-being appears to be a critical issue among minorities under the changing living environments.

Nonetheless, inconsistencies exist in the current environment as observable positive changes that have occurred in both groups as a result of education, spiritual strength, awareness creations, and policy contents implemented by concerning organizations, including nongovernmental projects. In this context, a lack of understanding about behavioral change and psychological factors means that a knowledge gap exists and paves the way for the current study. How might scholars assess this? Do ethnic minorities experience ethnic or racial discrimination more or less often than before? Alternatively, were these discriminatory actions perceived?

These considerations led to the following hypothesis:

Hypothesis 1: The Manjo, a minority ethnic group, will report lower levels of psychological well-being than their majority group counterparts.

The study aims to understand how psychological health varies across ethnic groups. This focus was drawn from studies showing that heightened levels of perceived discrimination among minority groups may cause minority stress that leads to lower psychological well-being (Meyer, 2003; Dengechi et al., 2018). Despite several studies on psychological well-being, few statistically proven scientific studies have been conducted to determine whether the variation in well-being is significant between two independent ethnic groups.

\section{Theoretical Framework}

There are various approaches to conceptualize psychological well-being across various disciplines including Psychology. Some primary approaches to conceptualize well-being are discussed with the following thought. 
Prior to World War II, most conceptualizations of health were focused on the absence of disease and disability. In 1948, however, the World Health Organization (WHO) proposed a definition that viewed health as "a state of complete physical, mental, and social well-being and not merely the absence of disease and infirmity" (WHO, 1948). Nonetheless, most health care research and practice continued to rely on the traditional medical model that focused on reducing disease and disability, with little attention given to the nature of health and well-being. The medical model was very useful for developing effective treatments for many illnesses but fell short in addressing the growing body of research that suggested the absence of pathology does not necessarily correlate with positive dimensions of health and well-being(Keyes, 2002 cited in Cooke et al., 2016). In most common thoughts, these varying conceptualizations can be categorized into four broad approaches.

The two most influential approaches in psychology have been the hedonic and eudaimonic schools (Deci\& Ryan, 2008). The hedonic approaches to conceptualizing well-being focus on pleasure and happiness (Deci\& Ryan, 2008). The most prominent hedonic model is known as subjective well-being, a tripartite model consisting of satisfaction with life, the absence of negative affect, and the presence of positive affect (Diener et al., 1985). Proponents of this perspective tend to conceptualize well-being in terms of all three of these constructs. The eudaimonic approaches to conceptualizing well-being suggest that psychological health is achieved by fulfilling one's potential, functioning at an optimal level, or realizing one's true nature (Lent, 2004). In contrast to the focus on affect and life satisfaction in the hedonic models, eudaimonic models tend to focus on a larger number of life domains, although they vary significantly regarding the fundamental elements that determine well-being. For example, one of the more prominent eudaimonic models is the psychological well-being model (Ryff, 1989; Ryff\& Keyes, 1995), which suggests that wellbeing consists of six elements: self-acceptance, positive relations with others, autonomy, environmental mastery, purpose in life, and personal growth. The eudaimonic model proposed by Deci and Ryan (2008), however, suggests that well-being is found in the fulfillment of three basic psychological needs: autonomy, competence, and relatedness. Clearly these two models overlap, but they also illustrate the variation found within the eudaimonic approaches to understanding wellbeing. A third category of approaches to conceptualizing well-being focuses on quality of life (QoL). The term QoL is often used interchangeably with wellbeing in the literature. For example, the authors who developed the Quality of Life Inventory use the terms quality of life, subjective well-being, and life satisfaction interchangeably (Frisch et al., 1992). However, those studying QoL generally conceptualize well-being more broadly than either the hedonic or eudaimonic models and include physical, psychological, and social aspects of functioning. This approach has been influenced by a variety of disciplines including medicine, sociology, and psychology.

A fourth category of conceptualizations of well-being is often referred to as wellness. Wellness approaches are rooted in the counseling literature and tend to be broader and less clearly defined than the approaches mentioned earlier (Roscoe, 2009). Similar to the situation for QoL, some authors use the term wellness interchangeably with well-being (Harari et al., 2005; Myers et al., 2004). One early definition of wellness shares with eudaimonic approaches a focus on optimal functioning and defines wellness as "an integrated method of functioning which is oriented toward maximizing the potential of which the individual is capable" (Palombi, 1992 as cited in Cooke et al., 2016). These four categories of approaches to understanding well-being have substantial similarities, with the broadest commonality being each construct's foundational interest in the positive dimension of human experience and functioning. Each category attempts to identify what constitutes "the good life" or optimal functioning for the human person (Deci\& Ryan, 2008) even 
if they differ on the particular terms used on the components of well-being, or the preferred measurement approach to operationalize well-being.

Based on the above theoretical grounds, this study's variables were conceptualized to compare the difference (statistical) between the two ethnic groups (Manjo minorities and the majority counterpart). This literally defined independent variable (ethnicity) and dependent variable (psychological well-being with six dimensions) were the bases to customize our understanding of this study as indicated in the following Figure 1:

\section{Figure 1}

\section{Conceptual Framework}

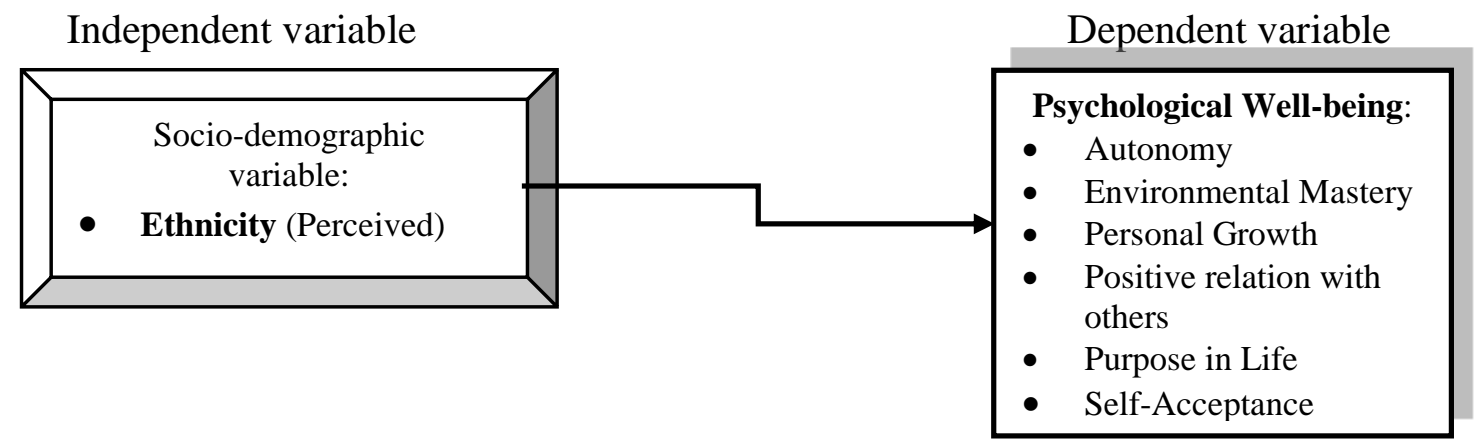

Moreover, Counseling research with Multicultural context in psychology offers a foundation for understanding internal processes and mental health outcomes for racial and ethnic minority groups (Hall, 2014). Despite the arrival of Manjo Ethnic Minorities in Kafficho (majority counterpart) for a long century, the literature still remains inadequate in pointing out what the perceived discrimination and psychological wellbeing (mental health outcomes) for these groups. In general, the studies that were conducted on the issues of Manjo Ethnic Minorities didn't indicate their self-perception and related psychological conditions. Therefore, in order to fill these gaps the current study was attempted to compare the psychological well-being of ethnic Manjo group with non-Manjo.

\section{Methods and Materials}

\section{Sampling and Population}

The target populations of this study were individuals from both Manjo ethnic minority group and non-Manjo group. At this time, based on the projection of Central Statistical Authority [CSA] 1994 census, the total population of Kaffa zone in 2007 was estimated about 100,679 particularly in the study area in Gimbo woreda (CSA, 2007). Even though the accurate number of Manjo ethnic group is not yet identified, predominantly in these three study kebeles, there are about 1,183 total households. Among this, 348 households were ethnic minority Manjo group and the rest 835 households were non-Manjo. Based on these household survey, a total of 298(149 Manjo and 149non-Manjo) study participants were drawn from two independent ethnic groups in the Kaffa zone, Gimboworeda. The sampling method was the cluster technique, which involves dividing the accessible population into clusters based on districts and ethnicity that would be subsequently picked in a simple random manner. This sampling method in the study was used 
because most of the Manjo live in clusters in areas separate from the majority Gomero. See Figure 2.

Figure 2

Stages of Sample Selection

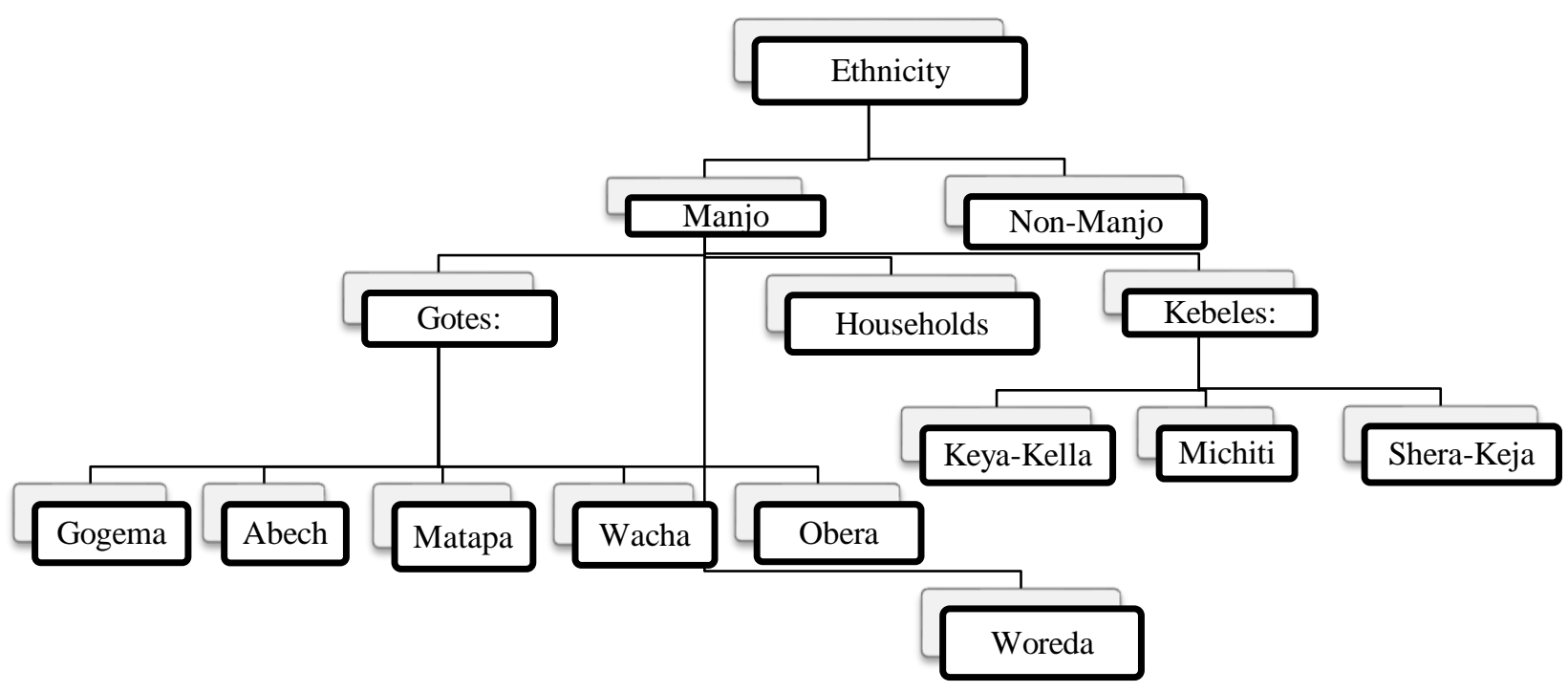

\section{Design}

This study used two types of statistics. First, descriptive statistics, including measures of central tendency and measures of spread, were used. Second, inferential statistics were applied to make generalizations about the populations from which the samples were drawn. Typically, in psychological studies, the investigator collects data and subsequently performs descriptive and inferential statistics. This was mainly due to a researcher might compute group means and use the null hypothesis significance testing procedure to draw conclusion about the population from which the groups were drawn. The same is true here a researcher emphasized these quantitative designs just because of the study nature and the researcher's intention to unfolding the value of numbers to explain facts. By some means, the study is also descriptive since it has attempted to measure the respondent's level of psychological well-being in the existing social phenomenon across two different groups. To this end, a big hunk of data with summary charts and tables presented, but do not attempt to draw conclusions about the sample population. Conversely, with inferential statistics, those sound inferential concepts like ANOVA and T-test were elaborated.

\section{Measurement}

The questionnaires for the survey used in this study were divided into two sub-sections. One part contained questions assessing the respondents' demographics, and the second part contained 42 items from Ryff's Psychological Well-Being Scales (PWB). This questionnaire that Ryff(1989) developed is divided into six subscales. Including 1) self-acceptance, 2) positive relationship with others, 3) autonomy, 4) environmental mastery, 5) purpose in life, and 6) a sense of personal growth. Seven items were designed to measure each subscale on a 6-point Likert scale 
with possible responses ranging from 1 (strongly disagree) to 6 (strongly agree). This scale also includes both positive and negative statements to look at the sample responses' consistency for each subscale. Negative items were reverse coded into positive ones, and the respondents with high scores were supposed to possess high psychological well-being and vice versa.

\section{Procedures of Data Collection}

After clearly explaining the study's purposes and registering the professional ID of the author with formally assigned research assistance so that he could work with communities near his institution, the researcher sought collectors for data collection. Data collectors were selected from personnel who had work experience and maturity because issues were potentially sensitive, especially with Manjo ethnic minorities. Thus, counselors, community social workers, and some teachers were chosen. First, they gathered data from the Manjo group. Then they went to the nonManjo group and gathered data. Much of questionnaires were administered while both Manjo and non-Manjo groups were having their weekly regular meeting in each selected districts. And these meetings were held under the two representatives independently. Then, they facilitated for the researcher to collect the data immediately after they finished their meeting. Additionally, there was also a door to door data gathering to meet those who did not come to the meeting. This was accomplished with the help of these two chairpersons. Before administering the instrument, the researcher informed the meeting participants who are previously selected and coded households for this study purpose and their rights. To this end, the data collectors were able to remove misunderstandings and suspicions that might arise from potential participants.

\section{Ethical Considerations}

In conducting this study, the following ethical considerations and safety measures were completed.

- Informed consent: The purposes and importance of the study were clearly explained to the participants of the study, and informed consent was obtained from each of them.

- Participation in the study was voluntary: Potential participants could refuse to participate or withdraw at any time.

- Privacy and confidentiality: Participants were informed that whatever information they provided would be kept confidential.

\section{Methods of Data Analysis}

The data from the research instrument were quantitatively analyzed. Descriptive analysis, including frequencies, percentages, means, and standard deviations, were employed to determine participants' levels of psychological well-being. ANOVA was conducted to examine the differences between the participants of ethnic groups in their level of psychological well-being. 


\section{Results}

\section{Preliminary Data Analysis}

The study samples were independently and randomly selected. Moreover, the data were checked for their normality of distribution using a box plot. See Figure 3.

\section{Figure 3}

Box Plot for the Normality of the Data Distribution

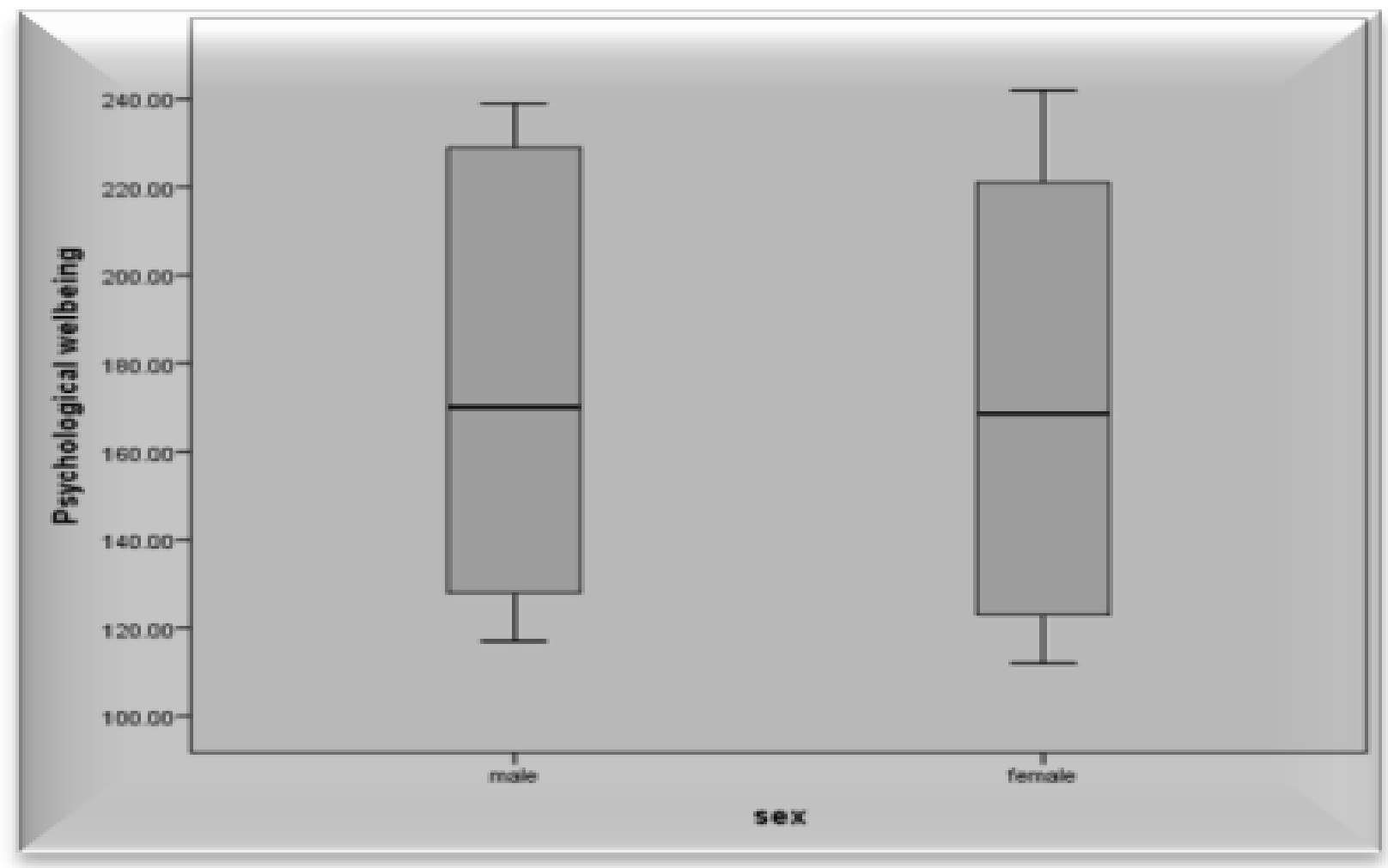

The preliminary check shown in Figure 3 indicated that the distribution of the psychological well-being across the two independent groups was fairly consistent with a normal distribution. The median was found close to the center of the box, and the whiskers were of approximately equal length, which suggested normal distribution.

The groups should come from populations with equal variances. To test this assumption, Levene's homogeneity of variance test was run in the Statistical Package for Social Sciences (SPSS20); Levene's test was considered for its significances, as indicated Table 1. The test shows that equality of variance in the measure of psychological well-being across two ethnic groups was significant [t (296), $\mathrm{p}<0.001]$.

\section{Table 1}

Test of Homogeneity of Variances

Psychological well-being

\begin{tabular}{rlll}
\hline Levene Statistic & df1 & df2 & Sig. \\
\hline 92.611 & 1 & 296 & .000 \\
\hline
\end{tabular}




\section{Demographics Distribution}

A total of 298 respondents were randomly selected to participate in this study. As shown in Table 2, there were three purposively selected kebele (district) and five specific gotes (subdistricts). Of the 298 total sample size, 26 (8.7\%) male, 27 (9.06\%) female respondents from the Manjo group; 26(8.7\%) male and 30(10.06\%) female participants of non-Manjo (Gomero) were selected from Keya-kellakebele, which involved two sub gotes (Gogema and Abech). 20 (6.7\%) male and 19 (6.3\%) female participants from Manjo; 17 (5.7\%) male and 18 (6.04\%) female participants from non-Manjo were selected from Michitikebele that involved one gote (Matapa); $28(9.39 \%)$ male and $29(9.73 \%)$ female participants from Manjo; 27 (9.06\%) male and 27(9.06\%) female respondents were selected from Shera-keja, which involved two sub-districts (Obera and Wacha).

Table 2

Summary of Background Information of Study Participants by Ethnicity, Gender and District Strata

\begin{tabular}{lcccccccc}
\hline KebelesGotes/Districts & \multicolumn{3}{c}{ Manjo } & \multicolumn{3}{c}{ Non-Manjo (Gomero) } \\
\hline & \multicolumn{2}{c}{ Mal } & \multicolumn{2}{c}{ Female } & \multicolumn{2}{c}{ Male } & \multicolumn{2}{c}{ Female } \\
\hline KeyakellaGogema & $\mathrm{N}$ & $\%$ & $\mathrm{~N}$ & $\%$ & $\mathrm{~N}$ & $\%$ & $\mathrm{~N}$ & $\%$ \\
Abech & 11 & 14.9 & 12 & 16.0 & 14 & 18.9 & 15 & 20.0 \\
MichitiMatapa & 15 & 20.3 & 15 & 20.0 & 16 & 21.6 & 15 & 20.0 \\
Wacha & 20 & 27.0 & 19 & 25.3 & 17 & 23.0 & 18 & 24.0 \\
SherakejaObera & 17 & 23.0 & 17 & 22.7 & 14 & 18.9 & 15 & 20.0 \\
\multicolumn{1}{c}{ Total } & 11 & 14.9 & 12 & 16.0 & 13 & 17.6 & 12 & 16.0 \\
& 74 & 100 & 75 & 100 & 74 & 100 & 75 & 100 \\
\hline
\end{tabular}

Descriptive Summary of Psychological Well-being in Manjo and non-ManjoGroup; N(Manjo=149; Gomero=149)

Concerning the overall level of psychological well-being, the mean level was in the high category in non-Manjo/Gomero $(\mathrm{M}=211.3, \mathrm{SD}=17.5)$, whereas it was in the low category (M $=158.2, \mathrm{SD}=39.6$ ) for Manjo Minorities, Compared to non-Manjo. See Table 3 below.

Table 3

Inter Group Statistics

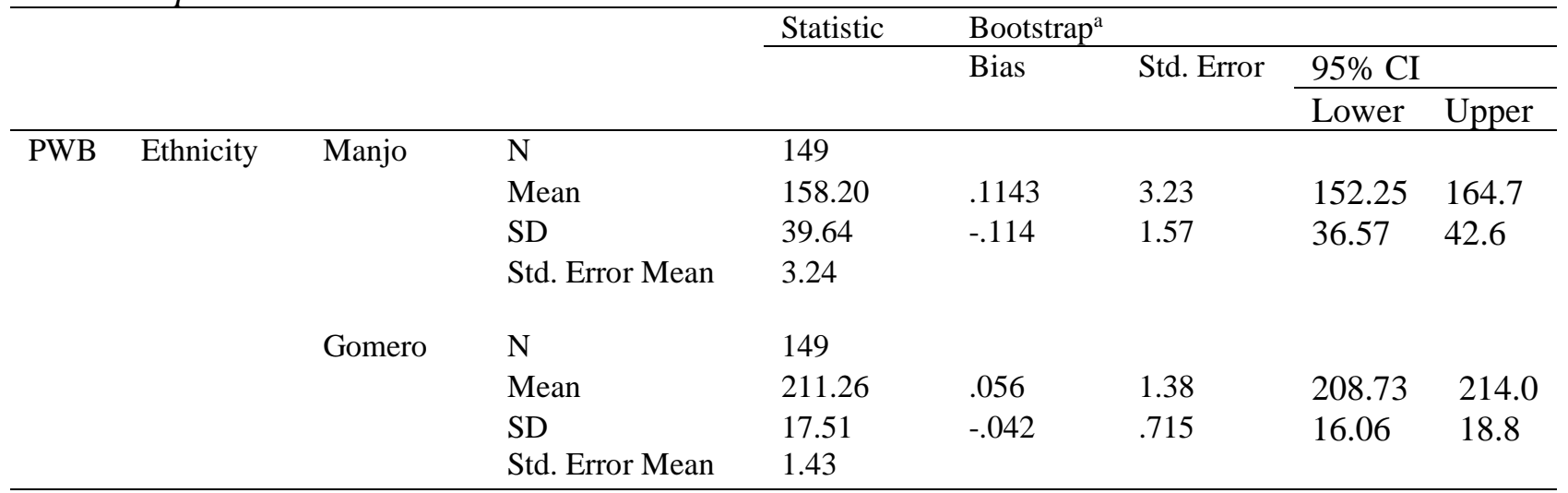

Note.PWB: Psychological well-being, CI: Confidence Interval. 


\section{Level of Psychological Well-being Along with Six Dimensions Across Groups}

Descriptive analysis was used to determine the level and pattern of the psychological wellbeing of groups on six of its dimensions (self-acceptance, positive relations with others, autonomy, environmental mastery, purpose in life, and personal growth). The findings indicated high score on the dimension of environmental mastery $(\mathrm{M}=41.1, \mathrm{SD}=4.2)$, followed by autonomy $(\mathrm{M}=39.7$, $\mathrm{SD}=4.6)$ for Gomero/non-Manjo/ group. On the other hand, the self-acceptance well-being dimension was higher for the Ethnic Minority Manjo group, where $(\mathrm{M}=28.2, \mathrm{SD}=8.2)$, followed by environmental mastery $(\mathrm{M}=27.7, \mathrm{SD}=10.9)$. The comparison was made not in between the two groups' level of mastery; rather, it looks within the group status along with the six dimensions. The lowest possible score attained for Manjo and non-Manjo was personal growth $(\mathrm{M}=25.0, \mathrm{SD}$ $=2.1$ and $\mathrm{M}=22.5, \mathrm{SD}=4.8$ ), respectively. See Table 4 below.

\section{Table 4}

Level of Psychological Well-being Dimensions Along with Six Dimensions

\begin{tabular}{|c|c|c|c|}
\hline & Ethnicity & & \\
\hline \multirow{10}{*}{ Autonomy } & Manjo & $\mathrm{N}$ & 149 \\
\hline & & Mean & 28.8054 \\
\hline & & Std. & 11.41045 \\
\hline & & Deviation & \\
\hline & & Std. Error & .93478 \\
\hline & Gomero & $\mathrm{N}$ & 149 \\
\hline & & Mean & 39.6846 \\
\hline & & Std. & 4.65003 \\
\hline & & Deviation & \\
\hline & & Std. Error & .38095 \\
\hline \multirow{11}{*}{ Environmental mastery } & Manjo & & \\
\hline & & $\mathrm{N}$ & 149 \\
\hline & & Mean & 27.7987 \\
\hline & & Std. & 10.97046 \\
\hline & & Deviation & \\
\hline & & Std. Error & .89874 \\
\hline & Gomero & $\mathrm{N}$ & 149 \\
\hline & & Mean & 41.1812 \\
\hline & & Std. & 4.23316 \\
\hline & & Deviation & .34679 \\
\hline & & Std. Error & \\
\hline \multirow[t]{11}{*}{ Personal growth } & Manjo & & 149 \\
\hline & & $\mathrm{N}$ & \\
\hline & & Mean & 22.4765 \\
\hline & & Std. & 4.85824 \\
\hline & & Deviation & \\
\hline & & Std. Error & .39800 \\
\hline & Gomero & $\mathrm{N}$ & 149 \\
\hline & & Mean & 25.0067 \\
\hline & & Std. & 2.11333 \\
\hline & & Deviation & \\
\hline & & Std. Error & .17313 \\
\hline Positive relation with other & Manjo & $\mathrm{N}$ & 149 \\
\hline
\end{tabular}




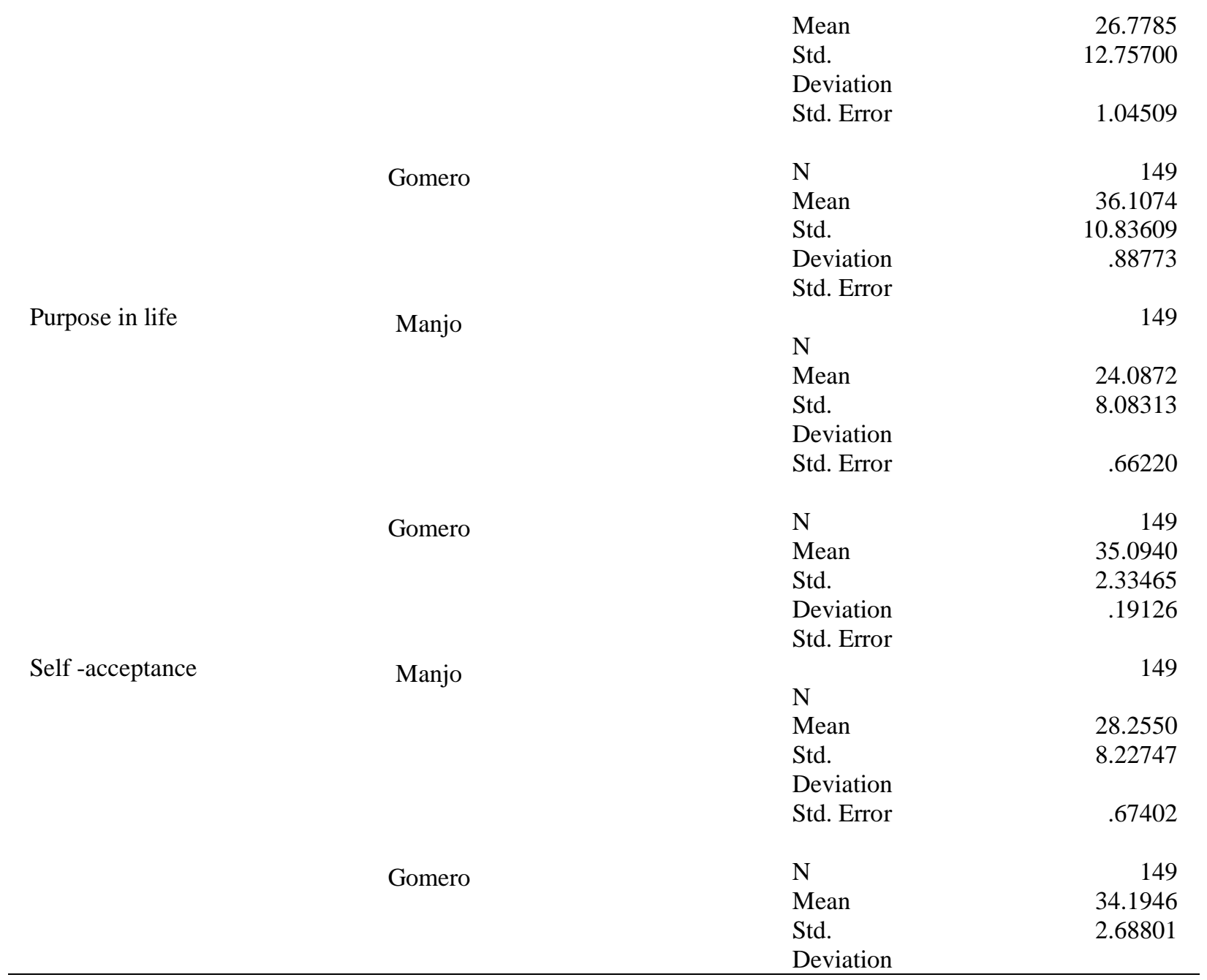

\section{The Difference in Psychological Well-being Across Ethnic Groups}

The mean variation (d) was -53.06, and an independent sample t-test was $-14.95 ; \mathrm{t}(296)$; $\mathrm{p}<0.01$, which was found significant. This finding implies that psychological well-being has a significant actual difference between the two study groups. See table 5 below.

Moreover, the effect size in mean difference $\left(\omega^{2}\right)$ was calculated using Cohen's idea that was a simple way of quantifying the difference between two groups that has many advantages over statistical significance tests alone. For the independent samples T-test, Cohen's ' $d$ ' is determined by calculating the mean difference between the two groups and dividing the result by the pooled standard deviation. Therefore, 0.58 was obtained for this current study sample, which was found to be $58 \%$ of variation explained in the dependent variable (Ethnicity) could be attributed to the difference between the two groups. Thus, 0.58 represents a medium effect size (Cohen \& Cohen, 1983). This means that the mean scores of the two groups differed by 0.58 standard deviations, and the difference was moderate even if it was statistically significant. 
Table 5

Independent Samples Test

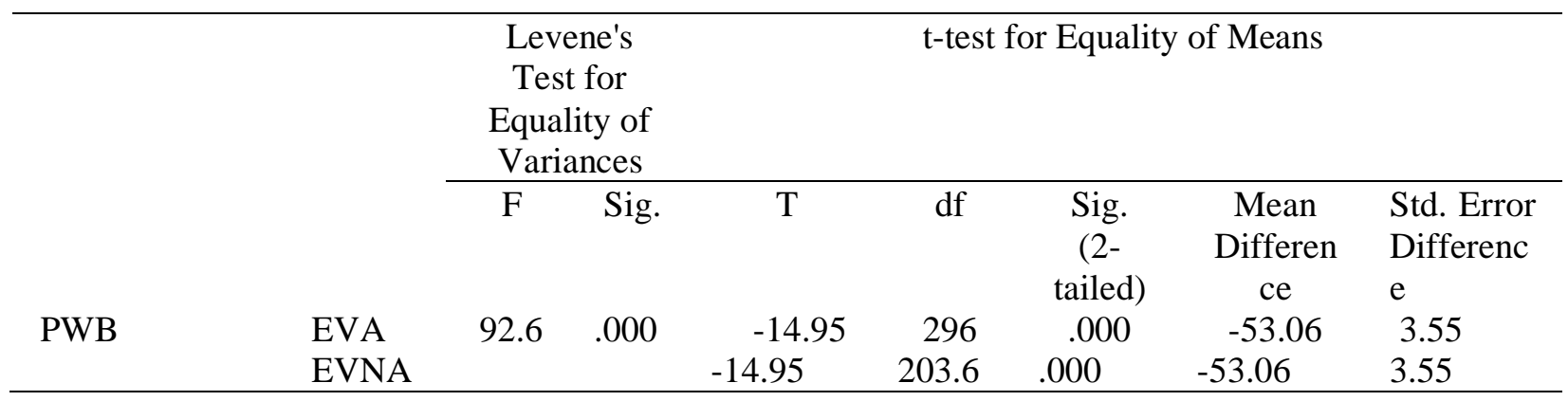

Note. PWB: Psychological well-being, EVA: Equal variances assumed, EVNA: Equal variances not assumed.

\section{Discussion}

Based on many reviews and searches including psycInfo search for 'ethnic' or 'ethnicity', 'culture' or 'cultural', and 'mental health' or 'psychological distress' or 'psychological wellbeing', the wide range of theoretical and empirical works have been added to this psychological study and used to supplement a report. Moreover, several studies have been done to describe the link between culture-related phenomena and mental health among different racial and ethnic minority groups in the United States and other Western countries. But this study has focused on the Eastern country's cultural and ethnic value through comparing the psychological well-being between Manjo ethnic minority group and the majority counterpart non-Manjo or Gomero in Ethiopia, Africa.

\section{Respondents' Level of Psychological Well-being}

The descriptive analyses indicated that the non-minority group possessed rather high levels of overall psychological well-being. Nevertheless, the dimension with the highest mean score was environmental mastery followed by autonomy, positive relations with others, purpose in life, and self-acceptance. In this context of the study, as ethnic minorities are required to deal with challenges either perceived or actual continually, they are unable to experience a higher level of Psychological health along with the overall well-being dimensions. In support of this claim, Williams et al. (2003) concluded that consistent findings show that "perceptions of discrimination tend to be associated with poorer health across a broad range of outcomes and across socially disadvantaged groups in different societies" (p.129-131). Among the 53 empirical studies, six reported the effects on subjective well-being measures like happiness and life satisfaction, and five of these assessed negative impact. The consequences of discrimination are similar to those of other stressors (James, 2019; Shieldset al., 2003; Wong et al., 2003).

Schaefer and Moos (1992) and Tedeschi and Calhoun (1996) also stated that growth is attained by retaining constant adaptability when encountering life crises or traumatic events as individuals who have encountered traumatic events are also able to be self-governing in difficult situations. Others have found that the perceived level of social discrimination has negative and significant correlations with the level of psychological well-being in Manjo Ethnic Minorities (Dengechi et al., 2018). Also, a substantial body of literature has recognized how negative psychological well-being outcomes are unevenly distributed across the U.S. population, with 
minority groups having a disproportionate burden amount of social stressors and discrimination that increase their risk of compromised mental health (Brown et al., 2000; Kessler et al., 1999; Krieger, 1999; Meyer, 1995; Pascoe \& Richman, 2009). Other work, however, has suggested that minority groups may develop protective strategies to minimize the negative consequences of discrimination Crocker and Major (1989) suggested that some minority group members develop strategies that reduce the impact of stigma or discrimination on mental health. More stigmatized populations will be more likely to report discrimination and may learn to minimize or buffer against the negative effects of perceived discrimination through efforts to resist or reject stigma (Camp et al., 2002; Thoits, 2011).

This notion had been consistently investigated in the current study groups in which the finding stated that it was the perceived discrimination (more of psychological) that the ethnic minorities have been facing in their daily lifestyles was found highly registered. And the difference between the two ethnic groups was also significant (Dachachi et al., 2020).Normally, this study found out that psychological well-being appears to significantly differ with multiple dimensions of mental health among the population of key status categories (Manjo and Gomero). Most minorities indicated low levels of day-to-day psychological well-being, yet the effects of ethnic status attached to it were still alive. Moreover, this study's hypothesis that posited "Minority status groups (Manjo) would be more likely to report a low level of psychological well-being than their majority group counterparts" was supported by the higher levels of psychological well-being among non-Manjo group members. This result suggested that the perceived discrimination by minority group members could partially explain the link between minority status and mental health disparities (Schwartz \& Meyer, 2010). The empirical findings, therefore, prove that the Manjo minority group was significantly more likely to report low levels of psychological well-being across varied measurements than their majority status counterparts.

In comparison, the counterpart majorities of non-Manjo group have scored highest in most well-being dimensions could probably predict the lowest score in perceived ethnic discrimination than Manjo minority. This in the other way indicates that there can be a very rare case (almost none) to experience perceived ethnic discrimination in non-Manjo group in their day to day life basis. This supports the notion that identifying strongly with a stigmatized group intensifies perceived discrimination in relation to being a member of that social group (Crocker \& Major, 1989). The results were indicative of perceived ethnic discriminative behaviors in such a way that ethnic based negative assumptions, ethnic based less respect, receiving poorer service at restaurant/bars, called names or insulted due to ethnicity and some other thought patterns are evident(Crocker \& Major, 1989).In line with this, an in-depth cross-national analysis of the association between perceived discrimination and psychological well-being in the USA compared with South Africa could be informative. Both countries share a history of legally enforced White supremacy and endogamy, and racial inequality that endures into the twenty-first century (Williams et al., 2010). Data obtained in this comparative study indicates that there were large racial differences in psychological well-being (self-esteem and mastery) in South Africa, with Whites reporting higher levels of both of these psychological resources than non-Whites. On the other study conducted by Pieterse, et al., (2012), because of the pervasive nature of race, especially for racial minorities, it has been shown to have a strong relationship with perceived ethnic discrimination and psychological distress for this population.

To this end, patterns of differences in psychological well-being by ethnic background are straightforward at first sight. Hence, using the 'equal variance assumed' test, the t-test revealed that there was a significant statistical mean difference between two ethnic groups. This impression could gone consistent with one study conducted by Awosogba \& Taylor (2014, p. 60) in the theoretical and empirical literature focusing on Black immigrants in USA, that those who perceived 
discrimination coming from European-Americans reported higher frequency of perceived discrimination $(\mathrm{M}=14.774, \mathrm{SD}=4.674)$ than those who did not come from European Americans $(\mathrm{M}=11.93, \mathrm{SD}=5.284)$.

An extensive literature, much of it generated in the 1950s and 1960s, articulated the line of optimal human functioning. Included were views of self-actualization (Maslow 1968), maturity (Allport 1961), individuation (Jung 1933), life-span development (Erikson 1959), the fully functioning person (Rogers 1961), and positive mental health (Jahoda 1958) (as cited in Ryff \& Singer, 1996). These humanistic accounts emphasized the full growth of the individual and successful negotiation of challenges confronted in life, such as finding meaning and purpose, having a sense of mastery, and being capable of autonomous action (Ryff \& Singer, 1996). Drawing on points of these theoretical formulations, this study has obtained the data on the measure of psychological wellbeing among Manjo and non-Manjo Group.

Even though there was no a single study directly compared the psychological well-being of Manjo ethnic minority group with that of non-Manjoin our country context, this single study was found reliable with findings explored in the context of ethnic minorities.

\section{Conclusion}

This study found a statistically significant variation in psychological well-being across a broad spectrum of measurement units between the minority group (Manjo) and its majority counterpart (Gomero). Each of which reflected their psychological health. In light of the current findings, future studies could be conducted. Multiple theoretical perspectives (see Meyer, 1995; Ridgeway, 2011) have suggested that low-status groups share similar experiences of internalizing negative societal attitudes, identifying a similar pathway between discrimination and mental health even though the level in processes likely varies between groups. The current finding also suggests that although this difference is not the same for all well-being dimensions among minority status groups, there were adequate connections to deserve future research combining multiple samples across the groups to extend the body of research that considers these minority status groups discretely. More research is needed to uncover the many manifestations of cultural discrimination and its processes and understand how the psycho-social well-being of ethnic minorities would be maintained.

The social elements such as ethnic status labeling and social power might have miserable indications in psychological well-being in particular and socio-cultural adjustments in general. In the sample, those who experienced lower psychological well-being scores would also predict that they have less social power (e.g. low in positive relation with others) and more difficulty with socio-cultural adjustment (e.g. low in environmental mastery, low in personal growth). This also would show that the two ethnic groups are in some level of cultural disorientation and that is why they become differ in psychology. Additionally, even though they had some level of positive relation with the host community but they may perceive as they are judged by their ethnic status which was culturally rooted for a long period of labeling. So this may also contribute for their lowered psychological state. To sum up the majority counterpart (Gomero) had better psychological well-being (compared to Manjo group) but this doesn't push us to conclude they have better psychological adjustment in all sub-dimensions. Although differences found statistically significant across each psychological well-being dimensions, but still have slight variations in some components of the psychological well-being measurements between two ethnic groups. 


\section{Implications}

Based on major findings of the study, the following points are reflected:

- Nonetheless there exist affirmative action given by a government for the Manjo ethnic minority group; it should be comprehensively extended to the maximum development of cognizance in both ethnic classes by targeting the adverse psychological effects of minority status perception.

- As it has been observed during and after the research process, there were some psychosocial service provisions by non-governmental organizations in those study sites such as CVM Ethiopia (CatholicVoluntarily Missionaries) and Action Aid Ethiopia. Hence, issues of minorities need special life-style guidance and counseling programs. This is a specialized service which demands adequate training on the part of the counselors and or the social workers. It is therefore recommended that the addressed organizations and their projects should consider the possibility of recruiting qualified counselors and social workers.

- For schools around the community, running the school counseling service for growing minority children and students by incorporating life skill mastery trainings to raise their sense of self, autonomy and integration issues is very essential.

- Since the issue of securing equality in human being is the task of both local and regional government, accelerated integration of policies, programs and trainings should be supposed to be designed in regular basis. Extended education on basic human rights and equalities should deliberately be devised for both ethnic groups particularly in the rural communities.

- Overall, the psychological care is equal importance as that of other needs like providing food for basic sustenance. Hence, communities in the majority counterpart need to understand the emotional sensitivities of minorities and should promote love, respect, social role integrations and further considerations.

- Finally, there is also a need for future exhaustive qualitative studies to gain detailed understanding in answering the "how" and "why" of the current psychological state and experiences of Manjo ethnic minorities exist in their real world. Thus, there is a need for more research to demarcate the psychological conditions of Manjo minorities.

\section{Acknowledgments}

We thank all professionals who have commented on this article and the study participants (particularly these Ethnic Minority classes of the Manjo) for taking part in the study.

\section{Funding Statement}

The authors received no specific funding for this work - the authors' self-sponsored the study.

\section{Competing Interests}

The authors have no competing interests. 


\section{Authors' Contributions}

This first listed author made substantial, direct, and intellectual contributions to the work from conception and design, data analysis and interpretation, drafting the article, and looking over it critically for important research content. Together with the second listed authors, the first author edited and approved the version to be published, and were accountable for all aspects of the work in ensuring that questions related to the accuracy or integrity of any part of the work were appropriately investigated and resolved.

\section{References}

Ahmed, E. (2009). The psycho-social and educational challenges of students of Manjo ethnic groups [Master's thesis, Addis Ababa University].

Armsden, G.C., \& Greenberg, M.T. (1987). The inventory of parent and peer attachment: Individual differences and their relationship to psychological well-being in adolescence. Journal of Youth and Adolescence, 16(5), 427-454. https://doi.org/10.1007/BF02202939

Awosogba, O., \& Taylor, D. (2014). A 12-year content analysis of the Journal of Black Psychology (2000-2011): Implications for the field of Black psychology. Journal of Black Psychology, 40(3), 215-238. https://doi.org/10.1177/0095798413486157.

Biddle, S. J. H., \& Asare, M. (2011). Physical activity and mental health in children and adolescents: A review of reviews. British Journal of Sports Medicine, 45, 886-895 doi:10.1136/bjsports-2011-090185

Brown, K. W., \& Ryan, R. M. (2003). The benefits of being present: Mindfulness and its role in psychological well-being. Journal of Personality \& Social Psychology, 84(4), 822-831.

Brown, T.N., Williams, D.R., Jackson, J.S., \& Neighbors, H.W. (2000). 'Being Black and feeling blue': The mental health consequences of racial discrimination. Race \& Society, 2(2),1731. https://doi.org/10.1016/S1090-9524(00)00010-3

Camp, D.L., Finlay, W.M.L. \& Lyons, E. (2002). Is low self-esteem an inevitable consequence of stigma? An example from women with chronic mental health problems. Social Science \& Medicine, 55(5), 823-34. https://doi.org/10.1016/S0277-9536(01)00205-2

Central Statistical Authority (2007). Population and Housing Census of Ethiopia.

Cohen, J., \& Cohen, P. (1983).Applied multiple regression/correlation analysis for the behavioral sciences (2nd ed.). Erlbaum.

Cooke, P. J., Timothy, P., Melchert, T., Korey, S.A., \& Connor, N. (2016).Measuring well-being: A review of instruments. Journal of counseling psychology, 44(5) 730-757. https://doi.org/10.1177/0011000016633507.

Crocker, J., \& Major, B. (1989). Social stigma and self-esteem: The self-protective properties of stigma. Psychological Review, 96(4), 608-630.

Dachachi, B. D., Woyessa, N. W., \&Weldmeskel, F. M. (2020).Perceived discrimination difference between the Manjo minority and Non-Manjo ethnic group in Kaffa Zone, Ethiopia. Journal of Research, Society \& Development, 9(9).http://dx.doi.org/10.33448/rsd-v9i9.7386

Deci, E. L.\& Ryan, R. M. (2008).Hedonia, Eudaimonia, and well-being: An introduction. Journal of Happiness Studies, 9, 1-11.

Dengechi, B., Worku, N., \&Mikre, F. (2018).Correlation of perceived discrimination and psychological well-being among the Manjo ethnic minority in Kaffa Zone, Ethiopia. Journal of Multicultural \& Multireligious Understanding, 5(6). http://dx.doi.org/10.18415/ijmmu.v5i6.465 
Diener, E., Emmons, R., Larsen, R., \& Griffin, S. (1985). The satisfaction with life scale. Journal of Personality Assessment, 49, 71- 75.

Farm Africa. (2002). Bonga integrated participatory forest management and reproductive health project, Phase 2. Addis Ababa.

Ersoy, E., \& Uysal, R. (2018). Opinions of School Psychological Counselors on Giftedness and Gifted Students' Education. American Journal of Qualitative Research, 2(2), 120-142.

Frisch, M. B., Cornell, J., Villanueva, M., \&Retzlaff, P. J. (1992).Clinical validation of the Quality of Life Inventory.A measure of life satisfaction for use in treatment planning and outcome assessment. Journal of Counseling Psychology, 4(1), 92-101.

Hall, C. I. (2014). The evolution of the revolution: The successful establishment of multicultural psychology. APA Handbook of Multicultural Psychology, Vol. 1: Theory \& Research (318). https://doi.org/10.1037/14189-001.

Harari, M. J., Waehler, C. A., \& Rogers, J. R. (2005). An empirical investigation of a theoretically based measure of perceived wellness. Journal of Counseling Psychology, 52(1), 93-103.

James, W. Y. (2019). Imprint of Racism: White Adult Males' Transformational Experience from Racial Antipathy to Racial Reconciliation. American Journal of Qualitative Research, 3(1), 93-116. https://doi.org/10.29333/ajqr/5813

Kessler, R.C., Mickelson, K.D., Williams, D.R. (1999). The prevalence, distribution, and mental health correlates of perceived discrimination in the United States. Journal of Health \& Social Behavior, 40(3), 208-230. http://www.jstor.org/stable/2676349

Krieger, N. (1999). Embodying inequality: A review of concepts, measures, and methods for studying health consequences of discrimination. International Journal of Health Services.29(2), 295-352. https://doi.org/10.2190/M11W-VWXE-KQM9-G97Q

Lakey, B., \&Orehek, E. (2011). Relational Regulation Theory: A New Approach to Explain the Link Between Perceived Social Support and Mental Health. Psychological Review, 118(3), 482-495. DOI: $10.1037 / \mathrm{a} 0023477$

Lent, R. W. (2004).Toward a unifying theoretical and practical perspective on wellbeing and psychosocial adjustment. Journal of Counseling Psychology, 51(4), 482-509. https://doi.org/10.1037/0022-0167.51.4.482.

Mesfin, T. (2005).Root causes, factors and effects of discrimination of Manjo community in Kaffa Zone[Research Report]. Action Aid Ethiopia, Bonga.

Meyer, I. H. (1995).Minority stress and mental health in gay men. Journal of Health \& Social Behavior, 36(1), 38-56. http://www.jstor.org/stable/2137286

Meyer, I. H. (2003). Prejudice, social stress, and mental health in lesbian, gay, and bisexual populations: Conceptual issues and research evidence. Psychological Bulletin, 129(5), 674-697. https://doi.org/10.1037/0033-2909.129.5.674

Myers, J. E., Luecht, R. M., \& Sweeney, T. J. (2004).Reexamining theoretical and empirical models underlying the Wellness Evaluation of Lifestyle (WEL) and the five-factor WEL. Measurement \&Evaluation in Counseling and Development, 26, 194-208.

Olli, E., \& Olsen, B.K. (Eds.).(2006). Common measures for discrimination II, Exploring possibilities for combining existing data for measuring ethnic discrimination. Centre for Combating Ethnic Discrimination/Danish Institute of Human Rights, Oslo/Copenhagen.

Pascoe, E. A., \& Richman, L. S. (2009). Perceived discrimination and health: A meta-analytic review. Psychological Bulletin, 135(4), 531-354.https://doi.org/10.1037/a0016059

Pieterse, A. L., Todd, N. R., Neville, H. A., \& Carter, R. T. (2012). Perceived racism and mental health among Black American adults: A meta-analytic review. Journal of Counseling Psychology, 59(1), 1-9. 
Ridgeway, C.L. (2011). Framed by gender: How gender inequality persists in the modern world. Oxford University Press.

Roscoe, L. J. (2009). Wellness: A review of theory and measurement for counselors. Journal of Counseling \& Development, 87(2), 216-226.

Ryff, C. D., \& Keyes, C. L. (1995).the structure of psychological well-being revisited. Journal Personality \& social Psychology, 69, 719-27.

Ryff, C. D., \& Singer, B. H. (1996). Psychological well-being: Meaning, measurement, and implications for psychotherapy research. Psychotherapy Psychosomatics, 65, 14-23. https://doi.org/10.1159/000289026

Ryff, C.D. (1989). Happiness is everything, or is it? Explorations on the meaning of psychological well-being. Journal of Personality \& Social Psychology, 57(6), 1069-1081.

Ryff, C.D., \& Singer, B.H. (2008). Know the self and become what you are: A eudaimonic approach to psychological well-being. Journal of Happiness Studies, 9(1), 13-39. https://doi.org/10.1007/s10902-006-9019-0

Schaefer, J.A., \& Moos, R.H. (1992).Life crises and personal growth. In B. N. Carpenter (Ed.),Personal coping: Theory, research, and application(pp. 149-170). Praeger Publishers/Greenwood Publishing Group

Schwartz, S., \&Meyer, I.H. (2010). Mental health disparities research: The impact of within and between group analyses on tests of social stress hypotheses. Social Science \& Medicine.70(8),1-8. https://doi.org/10.1016/j.socscimed.2009.11.032

Shields, M., Stephen, A., \& Price, W. (2003).The labor market outcomes and the psychological well-being of ethnic minority migrants in Britain. Home Office Online Report 07/03.

Tedeschi, R.G., \& Calhoun, L.G. (1996). The Posttraumatic Growth Inventory: Measuring the positive legacy of trauma. Journal of Traumatic Stress, 9(3), 455-471. https://doi.org/10.1007/BF02103658

Thoits, P.A. (2011). Resisting the stigma of mental illness. Social PsychologyQuarterly.74(1), 628. https://doi.org/10.1177/0190272511398019.

Williams, D. R., Neighbors, H.W., \& Jackson, J.S. (2003). Racial/ethnic discrimination and health: Findings from community studies. American Journal of Public Health,93(2). https://ajph.aphapublications.org/doi/full/10.2105/AJPH.93.2.200

Williams, D.R., Herman, A., Stein, D.J., Heeringa, S.G., Jackson, P.B., Moomal, H., \&Kessler, R.C. (2009). Discrimination and racial disparities in health: Evidence and needed research. Journal of Behavioral Medicine, 32(1), 20-47. https://doi.org/10.1007/s10865-008-9185-0

Williams, D.R., Mohammed, S.A., Leavell, J., \& Collins, C. (2010). Race, socioeconomic status, and health: Annals of the New York Academy of Sciences (89-101). http://dx.doi.org/10.1111/j.1749-6632.2009. 05339.x.

Wong, C., Eccles, J.A., \&Sameroff, A. (2003). Ethnic discrimination and ethnic identification: The influence on African-American adolescents' school and social emotional adjustments. Journal of Personality, 76(6).https://doi.org/10.1111/1467-6494.7106012

World Health Organization. (1948). The World Health Organization. Quality of Life Assessment (WHOQOL):Development and general psychometric properties. Journal of Social Science\& Medicine, 46(12), 1569-1585.

Yoshida, S. (2008).Searching for a way out of social discrimination: A case study of the Manjo through the 2002 incident in Kaffa. Journal of Japan Association for Nilo-Ethiopia Studies, $12(1), 47-60$. 
Yoshida, S. (2013). The struggle against social discrimination: Petitions by the Manjo in the Kaffa and Sheka Zones of South West Ethiopia. Journal of Japan Association for Nilo-Ethiopian Studies, 18(1), 1-19.

\section{Notes on Contributors}

Bizuayehu Dengechi, MA, is a lecturer in Psychology at the Department of Psychology of Social Science and Humanity College at Miazan-Tepi University, Ethiopia. He holds his MA degree in counseling psychology from Jimma University, Ethiopia. His work focuses on teaching and learning, research and community services. He works and teaches primarily counseling psychology. He also delivers other psychological courses in the department. His research interests are culture and psychology, multicultural education, minorities and psychology and social work.

Nigatuwa Worku, MA, is a lecturer in Psychology at the College of Education and Behavioral Science, Department of Psychology in Jimma University, Ethiopia. Currently, she is delivering courses for graduate as well as undergraduate studies, supervising students' practical works and providing community services. Besides, she works with different research teams in various issues in the community and cross-cutting issues like gender and minorities.

Fisseha Mikre, Ph.D., is associate professor of Educational Psychology at the department of psychology in Jimma University, Ethiopia. He has served the department of psychology for the last seventeen years. The major tasks he accomplishes in the department are teaching courses such as psychological testing for undergraduate psychology, assessment and evaluation of student learning for teacher education majors, standardized tests and educational psychology for postgraduate master and doctoral level students. Moreover, he is involved in the supervision thesis works of master and doctoral level students. The major research areas he is interested are educational and psychological assessment, psychology of minority groups, and the use of stories in counseling and psychotherapy practice. 